REVIEW ARTICLE

I.Q. Grunwald

A.K. Wakhloo

S. Walter

A.J. Molyneux

J.V. Byrne

S. Nagel

A.L. Kühn

M. Papadakis

K. Fassbender

J.S. Balami

M. Roffi

H. Sievert

A. Buchan

\title{
Endovascular Stroke Treatment Today
}

SUMMARY: The purpose of this study was to review current treatment options in acute ischemic stroke, focusing on the latest advances in the field of mechanical recanalization. These devices recently made available for endovascular intracranial thrombectomy show great potential in acute stroke treatments. Compelling evidence of their recanalization efficacy comes from current mechanical embolectomy trials. In addition to allowing an extension of the therapeutic time window, mechanical recanalization devices can be used without adjuvant thrombolytic therapy, thus diminishing the intracranial bleeding risk. Therefore, these devices are particularly suitable in patients in whom thrombolytic therapy is contraindicated. IV and IA thrombolysis and bridging therapy are viable options in acute stroke treatment. Mechanical recanalization devices can potentially have a clinically relevant impact in the interventional treatment of stroke, but at the present time, a randomized study would be beneficial.

ABBREVIATIONS: CE = conformité européenne; DEDAS = Dose Escalation of Desmoteplase for Acute Ischemic Stroke; DIAS = Desmoteplase in Acute Ischemic Stroke; DWI = diffusionweighted imaging; ECASS = European Cooperative Acute Stroke Studies; EPITHET = Echoplanar Imaging Thrombolytic Evaluation Trial; IA = intra-arterial; IMS = Interventional Management of Stroke; IV = intravenous; MCA = middle cerebral artery; mRS = modified Rankin Scale; NIHSS = National Institutes of Health Stroke Scale; PROACT = Prolyse in Acute Cerebral Thromboembolism; PWI = perfusion-weighted imaging; rtPA = recombinant tissue plasminogen activator; $\mathrm{TIMI}=$ Thrombolysis in Myocardial Infarction

$\mathbf{S}_{\mathrm{d}}^{\mathrm{c}}$ everal new mechanical thrombectomy devices have been designed with the goal of improving the recanalization rates of major intracranial artery occlusions. A limiting factor of first-generation devices was the lack of flexibility of the delivering catheters and the large size of the introducer sheath, which hindered navigation in the intracranial vasculature. We describe a new generation of mechanical recanalization devices that have recently been developed either as alternative or front-line therapies.

\section{Thrombolysis}

It was only in 1996 that the United States Food and Drug Administration approved IV rtPA for the treatment of acute ischemic stroke on the basis of 2 randomized trials comparing the effects of IV rtPA with a placebo in patients treated within 3 hours of stroke-symptom onset. ${ }^{1}$ IV rtPA has been shown to improve 3-month outcome if administered within the 3-hour

\section{Received July 30, 2010; accepted after revision August 4}

From the Biomedical Research Centre (I.Q.G.) and Neurovascular Research Unit (I.Q.G., A.J.M., J.V.B.), Nuffield Department of Surgery, University of Oxford and Oxford Radcliffe Hospitals NHS Trust, and Acute Stroke Programme (S.N., M.P.), Laboratory of Cerebral Ischaemia, Nuffield Department of Clinical Medicine, and Nuffield Department of Medicine, Medical Sciences Division (A.B., I.Q.G., J.S.B.), University of Oxford, Oxford, UK; Department of Radiology (A.K.W.), University of Massachusetts, Worcester, Massachusetts; Clinic for Neurology (S.W., K.F.) Saarland University Clinic, Homburg, Germany, and Department of Neurology (S.N.), University of Heidelberg, Heidelberg, Germany; Department of Neurology (A.L.K.), Centre Hospitalier de Luxembourg, Luxembourg-City, Luxembourg; Division of Cardiology (M.R.), Department of Internal Medicine, University Hospital Geneva, Switzerland; Cardiovascular Center Frankfurt (H.S.), Frankfurt, Germany.

References for this review were identified by searches of PubMed from 1980 to 2010 with the terms "acute stroke," "mechanical recanalization," "endovascular," "thrombectomy," "intra-arterial," and "stroke trials." Articles were also identified through searches of the authors' own files. Only articles published in English were reviewed.

Please address correspondence to Iris Quasar Grunwald, MD, Acute Vascular Imaging Centre, Biomedical Research Centre, University of Oxford and Oxford Radcliffe Hospital NHS Trust, John Radcliffe Hospital, Oxford OX3 9DU, UK; e-mail: i.grunwald@web.de DOI 10.3174/ajnr.A2346

Indicates open access to non-subscribers at www.ajnr.org "critical window of opportunity" from stroke-symptom onset. ${ }^{1}$ However, the percentage of patients with ischemic stroke who indeed are treated with thrombolytic therapy is exceedingly low, ranging from $2 \%$ to $3 \%$ in the United States to $4 \%$ in Europe. ${ }^{2-4}$ The reasons for its poor adoption are primarily the narrow time window required from symptom onset to drug administration and the potential for intracranial hemorrhage. Effort has been directed at expanding the time window by using PWI and DWI of the ischemic penumbra as a marker, to optimize selection of patients who would still respond to therapy beyond the 3-hour window. The "mismatch" regions (areas of PWI abnormality that do not have corresponding DWI lesions) have been considered likely to benefit from reperfusion therapies. This hypothesis has been assessed in recent clinical trials, including DIAS, DEDAS, DIAS II, Diffusion and Perfusion Imaging Evaluation for Understanding Stroke Evolution, and now, most recently, EPITHET. ${ }^{5-9}$

In EPITHET, mismatch patients treated with tissue plasminogen activator had a nonsignificant trend toward good neurologic outcome compared with the placebo patients $(50 \%$ versus 37\%). The authors estimated that a sample size of 225 patients per group would likely be adequate to demonstrate significant differences in their primary outcome. Until recently, user-friendly postprocessing software has not been available. The failure to postprocess PWI maps can contribute to suboptimal agreement between local investigators and central reading committees regarding which patients actually have a mismatch. This was demonstrated in the DEDAS trial. ${ }^{7}$ Thirty-two percent of the patients enrolled were determined by the central imaging laboratory to have violated the MR imaging inclusion criteria. In half of these violations, the core laboratory could not confirm a mismatch.

Beyond rtPA, other thrombolytics have been studied to push the upper limits of the treatment time window while simultaneously attempting to limit the risk of hemorrhagic transformation by using scanning technology to determine 
the size of the ischemic penumbra. One such agent is desmoteplase, which is made from bat venom and is a longer acting thrombolytic with greater fibrin specificity than rtPA. This agent was used in the DIAS II trial, which attempted to extend the 3-hour time window for thrombolytic therapy by identifying at least $20 \%$ ischemic-yet-salvageable brain tissue (penumbra) by using DWI/PWI mismatch techniques 3-9 hours after stroke onset. The DIAS II comprised 186 patients who were randomized into a placebo-controlled dose-ranging trial investigating the efficacy and safety of 2 doses of desmoteplase, $90 \mu \mathrm{g} / \mathrm{kg}$ and $125 \mu \mathrm{g} / \mathrm{kg}$, given as an IV bolus. There was no significant difference in the 3 groups as to improvement on the NIHSS score. Mortality was $6.3 \%$ in the placebo group, $5.3 \%$ in the $90-\mu \mathrm{g} / \mathrm{kg}$ group, and $21.2 \%$ in the $125-\mu \mathrm{g} / \mathrm{kg}$ group. Symptomatic intracranial hemorrhage occurred in $0 \%, 3.5 \%$, and $4.5 \%$ of patients, respectively.

The overestimation of the true volume of critically hypoperfused tissue may also have contributed to the failure of the recently reported DIAS II trial to document benefits of the thrombolytic desmoteplase in mismatch patients. When the DIAS II data were analyzed with a more conservative definition of mismatch (PWI-DWI volume $>75 \mathrm{~mL}$ ), beneficial effects of the thrombolytic agent desmoteplase were apparent. ${ }^{9}$ A recently published meta-analysis ${ }^{10}$ showed that delayed thrombolysis among patients selected according to mismatch imaging was associated with increased reperfusion/ recanalization, which was associated with improved outcomes. The fact that delayed thrombolysis in mismatch patients did not improve clinical outcome might be due to the failure to postprocess PWI maps described above. Recent advances in penumbral imaging technology should now enable penumbral selection of patients for trials of acute stroke therapy; however, a validation of the mismatch-selection paradigm is required. ${ }^{10,11}$

Experimental studies have shown that large intracranial thrombi can be resistant to thrombolytic treatment; these findings emphasize the need for therapeutic alternatives. ${ }^{12}$ Current data suggest that $>50 \%$ of patients who receive rtPA do not have a favorable clinical response. ${ }^{13,14}$ In fact, when IV therapy is used beyond the 3 -hour window, there is an inherent risk of intracranial bleeding and cerebral edema from reperfusion injuries. ECASS I and II failed to demonstrate the efficacy of thrombolytic therapy when used $\leq 6$ hours. ${ }^{15,16}$ However, recent results from ECASS III showed that in 821 patients with stroke, those allocated to IV rtPA administered $\leq 4.5$ hours from symptom onset $(n=418)$ were more likely to have a favorable outcome at 90 days as defined by an $\mathrm{mRS}$ of $0-1$ (52.4\% versus $45.2 \%$ for placebo, $P=.04)$. While mortality rates were not significantly different $(7.7 \%$ versus $8.4 \%)$, intracranial hemorrhage was observed with a greater frequency in the treatment group $(27.0 \%$ versus $17.6 \%, P=$ $.001){ }^{17}$ The extension of the time window from symptom onset to thrombolytic drug administration from 3 to 4.5 hours is promising and has been demonstrated as safe in a real world setting, reaching even better results than in ECASS III. ${ }^{18}$

\section{Intra-Arterial Thrombolysis}

In an effort to extend treatment availability to patients with stroke and to reduce the potential for intracranial hemor- rhage, IA lysis is a viable option for those who present in the 3to 6-hour time window, independent of the degree of neurologic impairment. Advantages and disadvantages of IA lysis, IV lysis, and combined IA/IV lysis have been extensively discussed. ${ }^{19-23}$ Early initiation of IV therapy followed by IA lytics for dissolution of large clot burden may be more efficacious, even though large clot burden in vessels such as the internal carotid artery terminus or basilar artery can often be resistant to thrombolytic therapy. ${ }^{24-26}$

The PROACT II study aimed to demonstrate that using prourokinase 6 hours from symptom onset in patients with an MCA occlusion would be of benefit. One hundred eighty patients with angiographically proved MCA occlusion were randomized in a 2:1 ratio to IA prourokinase or heparin control. The study showed a 15\% absolute benefit in functional independence at 90 days (mRS score, 0-2) accruing to patients receiving treatment with prourokinase. Other studies have addressed the question of whether delivering a lytic agent directly into the thrombus increases recanalization rates and/or reduces bleeding complications. Unfortunately IA prourokinase did not receive US Food and Drug Administration approval. The Japanese Middle Cerebral Artery Embolism Local Fibrinolytic Intervention Trial aimed to determine the safety and clinical efficacy of IA infusion of urokinase in patients with stroke within 6 hours of onset. This randomized trial might have provided additional evidence for the efficacy of IA therapy but was stopped prematurely by the Independent Monitoring Committee after approval of IV rtPA in Japan. A total of 114 patients underwent randomization. Good clinical outcome (mRS score, $0-1$ ) was more frequent in the urokinase group than in the control group (42.1\% versus $22.8 \%$, $P=.045)$. Although mRS $0-2$ at 90 days was more frequent in the urokinase group $(49.1 \%$ versus $38.6 \%)$, this did not reach a significant level $(P=.345)$. However, there were significantly more patients with NIHSS scores of 0 or 1 at 90 days in the urokinase group than the control group $(P=.017)$.

A recent publication comparing the outcome measures of IA versus IV thrombolysis for ischemic stroke in 112 patients deserves comment. ${ }^{27}$ Two very similar groups of patients with MCA occlusion as detected by the hyperattenuated sign on CT were assigned to IV plasminogen activator or IA urokinase within 3 (IV) or 6 (IA) hours, respectively. Despite the fact that the mean time from symptom onset to treatment was longer (244 minutes) in the IA group than the IV group (156 minutes), patients in the IA group had a significantly better (mRS score, $0-2$ ) outcome (53\% versus $23 \%, P<.001)$. Furthermore, mortality in the IA group was lower $(n=4.7 \%)$ compared with the IV group $(n=13,23 \% ; P<.003)$. The authors concluded that IA thrombolysis in this specific group of patients with a hyperattenuated MCA sign is superior to IV lysis.

\section{Intravascular Thrombolysis Combined with Mechanical Thrombectomy}

Combination use of IA lytics and mechanical thrombectomy devices is increasing and may be successful when each single strategy failed. When IA lytics have been unsuccessful in lysing the clot, mechanical devices could successfully recanalize the occluded cerebral vessel and restore flow. The designs of the devices vary between their individual engineering concepts 
and their approaches to the clot, either proximally or distally. However, a significant concern is the risk of vessel penetration when the technique requires the interventionalist to navigate the embolectomy device distal to the clot.

Stroke treatment improved when mechanical devices first became available. Although they were initially only accessible to patients who were part of trials, endovascular stroke interventions combined the use of IA thrombolytics, mechanical embolectomy devices, and angioplasty with or without stent placement. These procedures were implemented in patients who were either refractory to or ineligible for intravenous rtPA.

A trial of fundamental importance is the IMS II Trial, ${ }^{28}$ which compared combined IV/IA lysis versus standard IV lysis in patients treated within the 3-hour window after symptom onset compared with an historical placebo-controlled NINDS study. ${ }^{1}$ Two-thirds of the IV rtPA standard dose was administered $(0.6 \mathrm{mg} / \mathrm{kg}, 15 \%$ of the dose as a bolus during 1 minute with the remainder administered during 30 minutes, up to 60-mg maximum). After angiography and in the presence of a persistent occlusion, an intervention was performed with IA therapy by using either the MicroLysUS infusion catheter (EKOS, Bothell, Washington) or a standard microcatheter. Of 3602 screened patients, 81 were enrolled and treated ( 26 with IV rtPA alone and 55 with IV/IA intervention). Of the 55 patients, 19 were treated with a standard microcatheter, and in 36 cases, IA lysis was combined with the EKOS microinfusion catheter. Up to $22 \mathrm{mg}$ of IA rtPA was administered during 2 hours or until thrombolysis was achieved. IMS II subjects had significantly better outcomes at 3 months than NINDS placebo-treated subjects for all end points (odds ratio, $\geq 2.7$ ) and better outcomes than NINDS rtPA-treated subjects as measured by the Barthel Index and global test statistics. IMS II $^{29}$ also provided evidence that the Primo (EKOS) sonography microcatheter exhibits a trend toward improved recanalization of the occlusion compared with a standard microcatheter, and it again confirmed the correlation between recanalization and reperfusion, with good clinical outcome and reduced mortality.

The randomized IMS III Trial, which started enrollment in 2006, compares the combined IV/IA approach with standard IV rtPA alone, within 3 hours of acute ischemic stroke onset. A secondary objective is to determine the cost-effectiveness of the combined IV/IA approach compared with standard IV rtPA. It includes not only the EKOS and standard microcatheters but also the Merci retriever (Concentric Medical, Mountain View, California) and the Penumbra System (Penumbra, Alameda, California) as potential options for clot removal in the IV/IA paradigm.

The recently published results of the RECANALISE study ${ }^{30}$ showed that a combined IV-endovascular approach was associated with higher recanalization rates than IV alteplase alone in patients with acute stroke and confirmed arterial occlusion. Fifty-three patients were treated with this dual approach, and recanalization was achieved in $87 \%$ versus $52 \%$ of 107 patients in the IV group (adjusted relative risk, 1.49; $95 \%$ confidence interval, 1.21-1.84; $P=.0002)$. Twenty-four-hour neurologic improvement (NIHSS score of 0 or 1 or an improvement of 4 points) occurred in $60 \%$ versus $39 \%$. Mortality rates (17\%) at 90 days did not differ in both groups, and symptomatic intra- cranial hemorrhage occurred in $9 \%$ in the IV-endovascular group and in $11 \%$ in the IV group. Better clinical outcome was associated with recanalization.

The successes, advantages, and risks of the various devices can be graded by their ability to recanalize to TIMI 2 or 3 , bleeding rates, prevention of clot fragmentation followed by distal embolization, and the ultimate test determined by the clinical outcome of the patient.

\section{The Merci Retriever}

One of the first devices was the Merci clot retriever (Concentric Medical). It is an IA-delivered corkscrew-shaped flexible nitinol (nickel titanium) wire that traverses and ensnares the thrombus, which is then removed by traction. The device consists of the Merci retriever, the Merci balloon guide catheter, and the Merci microcatheter (Concentric Medical). The Merci clot retriever was evaluated in an international multicenter prospective single-arm trial (Mechanical Embolus Removal in Cerebral Ischemia I) for intracranial occlusion in 28 patients presenting up to 8 hours after symptom onset. Successful recanalization was defined as achieving TIMI 2 or 3 flow in the treated vessels. This first prospective study that examined a device-based treatment option for ischemic stroke showed a $43 \%$ recanalization rate with the device alone and a $64 \%$ recanalization rate with additional IA rtPA. ${ }^{31}$ The Multi Merci study revealed a recanalization rate of $57.3 \%$ with the newgeneration L5 Retriever and 69.5\% after adjunctive IA therapy as proved by conventional angiography among 131 patients. ${ }^{26}$ The Multi Merci study further showed a notable $27 \%$ absolute difference in mortality between patients who were successfully recanalized versus those who were not, thus proving the reperfusion paradigm.

A more recent publication examined the efficiency and possible complications of 2 different thrombectomy methods, in an animal model. ${ }^{32}$ They used the Merci retriever system (Concentric Medical) and the Catch system (BALT Extrusion, Montmorency, France), a tiny wire basket that retrieves the thrombus. Both devices work at the distal end of the clot. The Merci retriever achieved a $90 \%$ recanalization success rate versus $70 \%$ with the Catch system. The authors concluded that the Merci system achieved faster recanalization with less distal emboli. No vessel perforation or dissections were reported for either device.

The breakthrough of mechanical recanalization, with or without concomitant lysis, is evident as technologies become more refined and sophisticated. Rapid restoration of blood flow to salvageable brain tissue should minimize brain damage and improve survival rates, thus improving clinical outcomes. ${ }^{33-35}$ Two mechanical recanalization devices evolved during the last year. These are the Phenox Clot Retriever (Phenox, Bochum, Germany) and the Penumbra System (Penumbra).

\section{The Phenox Clot Retriever}

The Phenox Clot Retriever consists of 2 embodiments: pCR (CE mark in 2006) and CRC (CE mark in 2008) (Fig 1B, -C). Both systems have a highly flexible core wire compound resembling a pipe cleaner with perpendicularly oriented polyamide microfilaments that create an attenuated palisade. The 


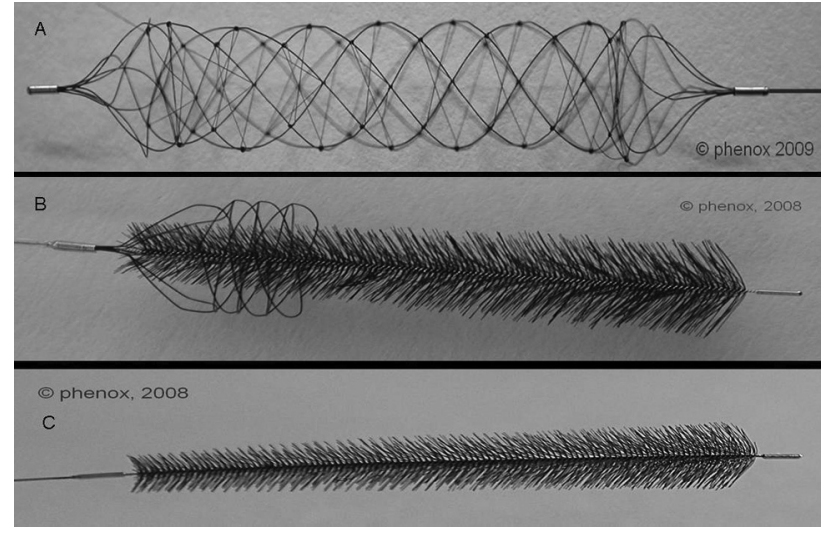

Fig 1. $A$, The BONnet consists of a self-expanding nitinol braiding with polyamide filaments passing through the interior to enlarge the surface area and enable better fixation of the thrombus mass. The system can be either put distal to the thrombus or released into the thrombus. $B$, The CRC is based on a fiber work of polyamide filaments whose lengths from proximal to distal end increase. The CRC has an additional nitinol thread cage at the proximal end of its fiber brush. This nitinol cage gives it a higher radial range. $C$, The Phenox pCR is based on perpendicularly oriented polyamide microfilaments that create an attenuated palisade.

proximal portion of the CRC is additionally surrounded by a nitinol wire braiding to offer more radial strength for mobilizing clots. The device is attached to the corpus of a microguidewire and comes in different sizes for vessels ranging from 1 to approximately $4 \mathrm{~mm}$ and can be inserted through a 0.021 or 0.027 -inch microcatheter, depending on device size. It is deployed distal to the clot and is slowly pulled back under continuous aspiration via the guiding catheter.

The BONnet (Phenox) (Fig 1A) was recently added to the 2 previous devices. It consists of a self-expanding nitinol braiding with polyamide filaments passing through the interior to enlarge the surface area and enable better fixation of the thrombus mass. The instrument is firmly connected with an insertion wire and carries at its proximal and distal ends $\mathrm{x}$-ray-visible markers. The system can be either put distal to the thrombus or released into the thrombus.

In comparative in vitro studies, the Phenox Clot Retriever was able to filter micro- and macrofragments that were formed during penetration and retrieval. ${ }^{36}$

\section{The Penumbra System}

The Penumbra System is based on an aspiration platform. This includes reperfusion microcatheters that are connected to an aspiration pump through aspiration tubing, generating a suction force of $-700 \mathrm{~mm} \mathrm{Hg}$. A teardrop-shaped separator is advanced and retracted within the lumen of the reperfusion catheter to debulk the clot for ease of aspiration (Fig 2).

As a nonpharmacologic tool, it has the potential of reopening a vessel without the use of adjunctive thrombolytics, thus offering dual options for recanalization via a single access platform. In addition, the system is designed to minimize the need to blindly penetrate into the occluded vascular segment because it operates from the proximal end of the clot. Due to the flexibility and variety of available sizes $(0.26-0.54$ inch $)$ of the reperfusion microcatheters, even smaller distal branches such as M2 and A2 can be successfully accessed for recanalization.

The first core laboratory-adjudicated feasibility phase 1 trial $^{37}$ showed a $100 \%$ revascularization rate to TIMI 2 or 3

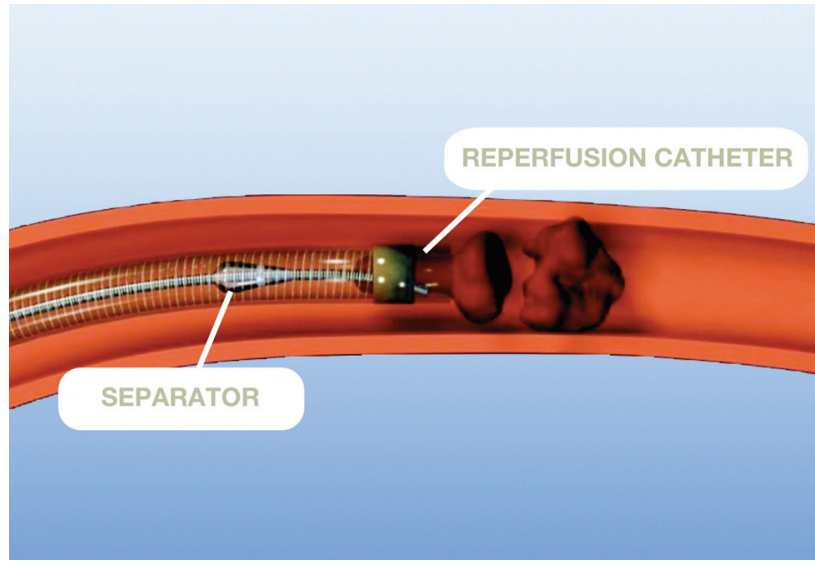

Fig 2. The Penumbra System is based on an aspiration platform that includes reperfusion microcatheters connected to an aspiration pump. A teardrop-shaped separator is advanced and retracted within the lumen of the reperfusion catheter to debulk the clot for ease of aspiration.

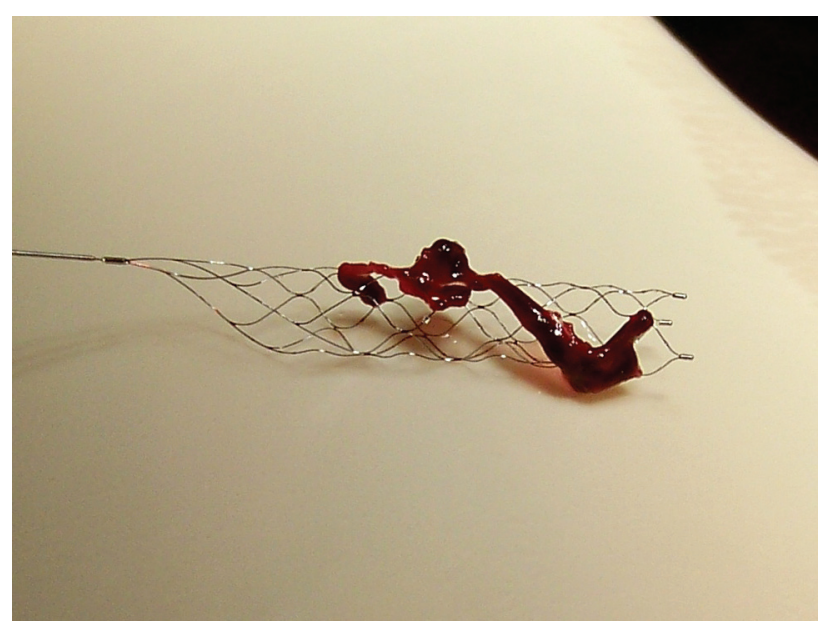

Fig 3. Solitaire FR stent (ev3). A self-expanding stent that can be fully deployed and then completely retrieved. It is not approved for sale in the United States.

among 20 patients ( 21 vessels). The pivotal phase 2 trial completed in September 2007 showed a TIMI 2 or 3 recanalization rate of $81.6 \%$ with a low procedural serious adverse event score of $3.4 \%$ in the 125 patients treated with the device; none were device-related. ${ }^{38}$ Symptomatic intracranial hemorrhage occurred in $11.2 \%$ of the patients, and all-cause mortality was $32.8 \%$. Good neurologic outcome as defined by a $>4$-point improvement on the NIHSS scores at discharge was observed in $57.8 \%$ of the patients, whereas good functional outcome as defined by a 90 -day mRS score of $0-2$ was reported in $25 \%$ of patients. There is evidence that patients who were successfully recanalized by the Penumbra System generally had better outcomes than those who were not. First single-center experiences as well as preliminary data from the first postmarket multicenter study seemed to confirm these findings. ${ }^{39-41}$ In a retrospective case review study in 139 patients treated by the Penumbra System at 7 centers in the United States and Europe, the device was found to successfully recanalize the target vessel in $84 \%$ of cases, with similar outcome and intracranial hemorrhage rates as those in the pivotal trial. In fact, $40 \%$ of the patients had achieved an mRS score of $<2$ at 90 days, which is a significantly higher rate than that reported earlier. 
Thus, it appears that the favorable safety and effectiveness profile of the Penumbra System observed in the pivotal trial can be extended to the real world setting.

\section{Stent Placement in Acute Stroke}

Recently the Solitaire stent (ev3, Plymouth, Minnesota) (Fig 3), a self-expanding stent that offers the unique capability of being fully deployed and then completely retrieved, if not detached, was used as a novel mechanical embolectomy device for large-artery occlusions (Image 3, ev3). The stent is not approved for sale in the United States yet but has received the CE mark. A single-center prospective pilot study ${ }^{42}$ on 20 patients with an acute ischemic stroke attributable to a large artery occlusion of the anterior circulation within the first 8 hours from symptom onset showed successful revascularization (Thrombolysis in Cerebral Infarction 2b or 3) in 18 of 20 $(90 \%)$ treated vessels. Forty-five percent of patients reached an mRS score of $\leq 2$ at 3 months.

\section{Conclusions}

The evolutionary journey of mechanical thrombectomy devices has traveled from adjunctive rescue treatment to frontline therapy. In essence, recanalization by using mechanical means could not only extend the therapeutic window but also potentially reduce the number of bleeds that are frequently associated with thrombolytic therapy. Limitations of mechanical devices have been reported and attributed to vessel tortuosity, arterial stenosis, and inaccessibility of the thrombus due to its location and consistency. The ongoing registries of the Merci clot retriever and the Penumbra System as well as the IMS III trial should, in the future, provide further evidence of these emerging technologies. Early consideration of this technique in patients with acute stroke secondary to large vessel cerebro-occlusive disease may improve recanalization rates and clinical outcomes.

\section{References}

1. NINDS rt-PA Stroke Study Group. Tissue plasminogen activator for acute ischemic stroke. N Engl J Med 1995;333:1581-87

2. Heuschmann PU, Berger K, Misselwitz B, et al. Frequency of thrombolytic therapy in patients with acute ischemic stroke and the risk of in-hospital mortality: the German Stroke Registers Study Group. Stroke 2003;34:1106-13

3. Schumacher HC, Bateman BT, Boden-Albala B, et al. Use of thrombolysis in acute ischemic stroke: analysis of the Nationwide Inpatient Sample 1999 to 2004. Ann Emerg Med 2007;50:99-107

4. Weimar C, Kraywinkel K, Maschke M, et al. Intravenous thrombolysis in German stroke units before and after regulatory approval of recombinant tissue plasminogen activator. Cerebrovasc Dis 2006;22:429-31

5. Albers GW, Thijs VN, Wechsler L, et al, for the DEFUSE Investigators. Magnetic resonance imaging profiles predict clinical response to early reperfusion: the Diffusion and Perfusion Imaging Evaluation for Understanding Stroke Evolution (DEFUSE) study. Ann Neurol 2006;60:508-17

6. Davis SM, Donnan GA, Parsons MW, et al, for the EPITHET Investigators. Effects of alteplase beyond $3 \mathrm{~h}$ after stroke in the Echoplanar Imaging Thrombolytic Evaluation Trial (EPITHET): a placebo-controlled randomised trial. Lancet Neurol 2008;7:299-309. Epub 2008 Feb 28

7. Furlan AJ, Eyding D, Albers GW, et al, for the DEDAS Investigators. Dose Escalation of Desmoteplase for Acute Ischemic Stroke (DEDAS): evidence of safety and efficacy 3 to 9 hours after stroke onset. Stroke 2006;37:1227-31

8. Hacke W, Albers G, Al-Rawi Y, et al, for the DIAS Study Group. The Desmoteplase in Acute Ischemic Stroke Trial (DIAS): a phase II MRI-based 9-hour window acute stroke thrombolysis trial with intravenous desmoteplase. Stroke 2005;36: 66-73

9. Warach S, for the DIAS-2 Study Group. Clinical benefit of desmoteplase treatment in patients with moderate to severe stroke: further results of the DIAS-2
Study. In: Proceedings of the International Stroke Conference, New Orleans, Louisiana. February 21, 2008

10. Mishra NK, Albers GW, Davis SM, et al. Mismatch-based delayed thrombolysis: a meta-analysis. Stroke 2010;41:e25-33. Epub 2009 Nov 19

11. Donnan GA, Baron JC, Ma H, et al. Penumbral selection of patients for trials of acute stroke therapy. Lancet Neurol 2009;8:261-69

12. Zivin JA, Fisher M, De Girolami U, et al. Tissue plasminogen activator reduces neurological damage after cerebral embolism. Science 1985;230:1289-92

13. Papadakis M, Buchan AM. Translational vehicles for neuroprotection. Biochem Soc Trans 2006;34:1318-22

14. Qureshi Al, Ali Z, Suri MF, et al. Intra-arterial third generation recombinant tissue plasminogen activator (reteplase) for acute ischemic stroke. Neurosurgery 2001;49:41-48, discussion 48-50

15. Hacke W, Kaste M, Fieschi C, et al. Intravenous thrombolysis with recombinant tissue plasminogen activator for acute hemispheric stroke: The European Cooperative Acute Stroke Study (ECASS). JAMA 1995;274:1017-25

16. Hacke W, Kaste M, Fieschi C, et al. Randomised double-blind placebo-controlled trial of thrombolytic therapy with intravenous alteplase in acute ischaemic stroke (ECASS II): Second European-Australasian Acute Stroke Study Investigators. Lancet 1998;352:1245-51

17. Hacke W, Kaste M, Bluhmki E, et al. Thrombolysis with alteplase 3 to 4.5 hours after acute ischemic stroke. $N$ Engl J Med 2008;359:1317-29

18. Wahlgren N, Ahmed N, Davalos A, et al, for the SITS Investigators. Thrombolysis with alteplase $3-4.5 \mathrm{~h}$ after acute ischaemic stroke (SITS-ISTR): an observational study. Lancet 2008;372:1303-09

19. IMS Study Investigators. Combined intravenous and intra-arterial recanalization for acute ischemic stroke: the Interventional Management of Stroke Study. Stroke 2004;35:904-11. Epub 2004 Mar 11

20. Flaherty ML, Woo D, Kissela B, et al. Combined IV and intra-arterial thrombolysis for acute ischemic stroke. Neurology 2005;64:386-88

21. Lee KY, Kim DI, Kim SH, et al. Sequential combination of intravenous recombinant tissue plasminogen activator and intra-arterial urokinase in acute ischemic stroke. AJNR Am J Neuroradiol 2004;25:1470-75

22. Lewandowski C, Frankel M, Tomsick T, et al, for the EMS Bridging Trial Investigators. Combined intravenous and intra-arterial r-TPA versus intra-arterial therapy of acute ischemic stroke: Emergency Management of Stroke (EMS) Bridging Trial. Stroke 1999;30:2598-605

23. Zaidat OO, Suarez JI, Santillan C, et al. Response to intra-arterial and combined intravenous and intra-arterial thrombolytic therapy in patients with distal internal carotid artery occlusion. Stroke 2002;33:1821-26

24. Del Zoppo GJ, Higashida RT, Furlan AJ, et al. PROACT: a phase II randomized trial of recombinant pro-urokinase by direct arterial delivery in acute middle cerebral artery stroke-PROACT Investigators. Prolyse in Acute Cerebral Thromboembolism. Stroke 1998;29:4-11

25. Furlan A, Higashida R, Wechsler L, et al. Intra-arterial prourokinase for acute ischemic stroke: The PROACT II study—a randomized controlled trial. Prolyse in Acute Cerebral Thromboembolism. JAMA 1999;282:2003-11

26. Smith W, Sung G, Saver J, et al. Mechanical thrombectomy for acute ischemic stroke: final results of the Multi Merci trial. Stroke 2008;39:1205-12. Epub 2008 Feb 28

27. Mattle HP, Arnold M, Georgiadis D, et al. Comparison of intraarterial and intravenous thrombolysis for ischemic stroke with hyperdense middle cerebral artery sign. Stroke 2008;39:379-83

28. The IMS II Trial Investigators. The Interventional Management of Stroke (IMS) II study. Stroke 2007;38:2127-35. Epub 2007 May 24

29. Tomsick T, Broderick J, Carrozella J, et al, for the Interventional Management of Stroke II Investigators. Revascularization results in the Interventional Management of Stroke II trial. AJNR Am J Neuroradiol 2008;29:582-87

30. Mazighi M, Serfaty JM, Labreuche J, et al. Comparison of intravenous alteplase with a combined intravenous-endovascular approach in patients with stroke and confirmed arterial occlusion (RECANALISE study): a prospective cohort study. Lancet Neurol 2009;8:802-09

31. Gobin YP, Starkman S, Duckwiler GR, et al. Merci 1: a phase 1 study of mechanical embolus removal in cerebral ischemia. Stroke 2004;35:2848-54

32. Brekenfeld C, Schroth G, El-Koussy M, et al. Mechanical thromboembolectomy for acute ischemic stroke: comparison of the catch thrombectomy device and the Merci retriever in vivo. Stroke 2008;39:1213-19. Epub 2008 Feb 28

33. Smith WS, Tsao JW, Billings ME, et al. Prognostic significance of angiographically confirmed large vessel intracranial occlusion in patients presenting with acute brain ischemia. Neurocrit Care 2006;4:14-17

34. Smith WS, Sung G, Starkmann S, et al. Safety and efficacy of mechanical embolectomy in acute ischemic stroke: results of the MERCI trial. Stroke 2005;36:1432-38

35. Smith WS. Intra-arterial thrombolytic therapy for acute basilar occlusion: pro. Stroke 2007;38:701-03

36. Liebig T, Reinartz J, Hannes R, et al. Comparative in vitro study of five mechanical embolectomy systems: effectiveness of clot removal and risk of distal embolization. Neuroradiology 2008;50:43-52

37. Bose A, Henkes H, Alfke K, et al, for the Penumbra Phase 1 Stroke Trial 
Investigators. The Penumbra system: a mechanical device for the treatment of acute stroke due to thromboembolism. AJNR Am J Neuroradiol 2008;29:1409-13

38. The Penumbra Pivotal Stroke Trial Investigators. The Penumbra pivotal stroke trial: safety and effectiveness of a new generation of mechanical devices for clot removal in intracranial large vessel occlusive disease. Stroke 2009;40: 2761-68. Epub 2009 Jul 9

39. Grunwald IQ, Bose A, Struffert T, et al. Images in neurology: liposuction in mind. Arch Neurol 2009;66:800-01
40. Grunwald IQ, Walter S, Papanagiotou P, et al. Revascularization in acute ischaemic stroke using the Penumbra system: the first single center experience. Eur J Neurol 2009;16:1210-16

41. Nogueira RG, Yoo AJ, Buonanno FS, et al. Endovascular approaches to acute stroke. Part 2. A comprehensive review of studies and trials. AJNR Am J Neuroradiol 2009;30:859-75

42. Castaño C, Dorado L, Guerrero C, et al. Mechanical thrombectomy with the Solitaire AB device in large artery occlusions of the anterior circulation: a pilot study. Stroke 2010;41:1836-40. Epub 2010 Jun 10 